\title{
Anterior EEG Asymmetry and the Modifier Model of Autism
}

\author{
Courtney P. Burnette $\cdot$ Heather A. Henderson • \\ Anne Pradella Inge $\cdot$ Nicole E. Zahka $\cdot$ \\ Caley B. Schwartz $\cdot$ Peter C. Mundy
}

Published online: 24 November 2010

(C) The Author(s) 2010. This article is published with open access at Springerlink.com

\begin{abstract}
Individual differences in the expression of autism complicate research on the nature and treatment of this disorder. In the Modifier Model of Autism (Mundy et al. 2007), we proposed that individual differences in autism may result not only from syndrome specific causal processes, but also from variability in generic, non-syndrome specific modifier processes that affect the social and emotional development of all people. One study supporting this model found that measures of resting anterior EEG asymmetry, a measure reflecting complex brain processes associated with generic individual differences in approach and avoidance motivation, may help explain differences in the expression of autism in children without intellectual disabilities (Sutton et al. 2005). In the current study, we partially replicated the observation that children with autism who exhibited a pattern of left frontal EEG asymmetry tended to display milder levels of social symptoms, although in the current sample this pattern applied only to HFA children with relatively lower verbal IQs. New observations indicated that left frontal EEG asymmetry was also associated with retrospective parent reports of significantly later age of onset of symptoms, but also higher levels of selfreported outward expressions of anger as well as symptoms
\end{abstract}

C. P. Burnette $(\square)$

University of New Mexico, Center for Development

and Disability, Albuquerque, NM, USA

e-mail: cburnette@salud.unm.edu

H. A. Henderson - A. P. Inge - N. E. Zahka - C. B. Schwartz Department of Psychology, University of Miami,

Coral Gables, FL, USA

P. C. Mundy

M.I.N.D. Institute and School of Education,

University of California at Davis, Davis, CA, USA of obsessive compulsive disorder in school-age higher functioning children with ASD. Therefore, the results of this study provide a new and fully independent set of observations, which indicate that individual differences in anterior EEG asymmetry may significantly moderate the expression and developmental course of autism. This observation may have clinical implications for identifying meaningful diagnostic sub-groups among children with autism.

Keywords EEG asymmetry - Social motivation · Individual differences

\section{Introduction}

Phenotypic variability in autism is commonly thought to be an outgrowth of variance in the biological Initial Causal Processes (ICPs) that give rise to this syndrome. According to the Modifier Model of Autism, though, additional sources of clinically meaningful variation in symptom expression and course may be associated with generic processes that are not specific to the etiology of autism. These generic Modifier Processes (MPs) contribute to differences in social, cognitive and emotional outcomes across typical development and many forms of psychopathology including autism (Mundy et al. 2007; see Fig. 1). MPs may interact with etiological processes to contribute to phenotypic variability in autism. Hence, identifying and understanding the effects of modifier processes is of high significance both clinically (Wood et al. 2009) and theoretically (Mundy et al. 2007; Henderson et al. 2006; Meyer et al. 2006; Sutton et al. 2005).

One long recognized axis of variability within autism is individual differences in approach and avoidance tendencies (Wing and Gould 1979). Many children with autism display 


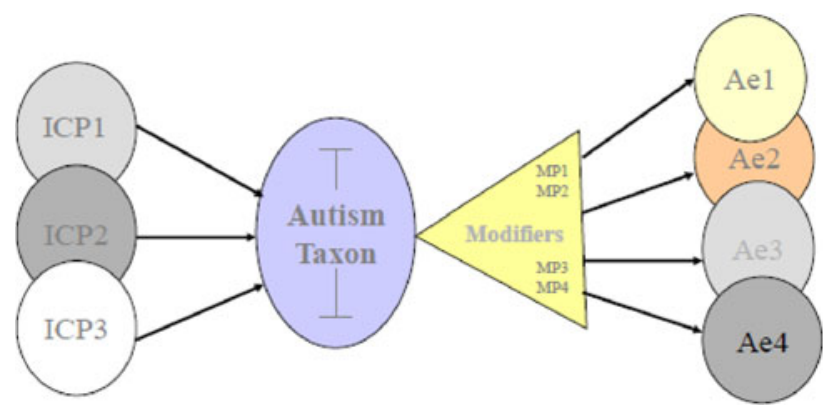

Fig. 1 Phenotypic variability in autism arises from two sources: Syndrome specific Initial Causal Processes $(I C P)$ and non-syndrome specific Modifier Processes $(M P)$. Different constellations of genetic and neurodevelopmental ICPs contribute to individual differences. In addition, there is a good deal of phenotypic variability in the expression of autism $(\mathrm{Ae} 1, \mathrm{Ae} 2, \ldots)$ that arise through interactions with non-syndrome specific MPs, such as approach and avoidance behavioral tendencies

reticent, avoidant, and/or inhibited behavior patterns. These children are less likely to initiate interactions with other people with some children characterized as "aloof" and others as "passive." In contrast, another group of children with autism is characterized by more demonstrative, active approach-oriented behavior patterns. These children are more likely to initiate more, albeit, maladroit interactions with others and consequently are referred to as "active but odd" (e.g., Wing and Gould 1979; O'Brien 1996). Previous data suggested that differences in aloof versus active but odd behavior may be associated with intellectual status, such that the active but odd group was more likely to include children with autism without intellectual disabilities (e.g., Volkmar et al. 1989). Earlier research also suggested that differences in frontal brain activity may be associated with the active but odd versus passive-aloof patterns of behavior in autism (Dawson et al. 1995).

More recently, individual differences in active-approach and passive-avoidant tendencies have been associated with differences in intervention responsiveness and developmental course in autism. Beglinger and Smith (2005) reported that aloof children with autism, ranging in age from 39- to 71-months, were less responsive to early intervention, in terms of cognitive gains, compared to active or passive children. Sherer and Schreibman (2005) also reported that five variables distinguished 36- to 45month olds with autism who had better responses to pivotal response training. The dimensions associated with positive intervention response included more approach and less avoidant behaviors. In a study of the early course of development of autism Garon et al. (2009) observed 34 of 138 infant siblings of children with autism who were ultimately diagnosed with an autism spectrum disorder. Sixteen of the children in this sample displayed relatively clear symptoms by 24 months, and 18 expressed clear symptom presentation later at 36 months of age. One of the primary distinguishing characteristics of these two subgroups of children was that the subgroup whom were older at the time of clear presentation were rated higher on behavioral approach at 24 months of age. Garon et al. (2009, p. 73) noted that the "striking parallel" of this finding with elements of the Modifier Model of Autism which together suggest that cortical systems involved in differences in active versus aloof behavior patterns may influence the clarity of symptom presentation in autism (Mundy et al. 2007).

Sutton et al. (2005) directly tested the hypothesis that individual differences in frontal EEG asymmetry may affect the expression of autism by examining the associations between resting anterior EEG asymmetry and symptom severity and social/emotional functioning in higher functioning children and adolescents with autism. The rationale for the Sutton et al. (2005) study was the extensive research literature demonstrating associations between approach behaviors and relatively more left than right resting frontal cortical activity and between avoidant behaviors and relatively greater right than left resting activation (e.g., Davidson 2002; Harmon-Jones and Allen 1998; Pizzagalli et al. 2005). Sutton et al. (2005) reasoned that if approach-avoidance tendencies modify the expression of autism, then measures of resting anterior EEG asymmetry, or default brain activity, may be expected to be associated with significant differences in symptom expression in autism. Consistent with this hypothesis, parent report on a symptom checklist indicated that 8- to 17-year-old higher functioning children with autism who displayed left frontal EEG asymmetry displayed fewer or less intense symptoms of social impairment than children with right frontal EEG asymmetry. Since left anterior EEG asymmetry is associated with approach behavior and right anterior asymmetry is associated with withdrawal, Sutton et al. (2005) interpreted their data to suggest the expression of social symptoms was milder, or less clear, in children with autism disposed toward approach behavioral tendencies. The data from Sutton et al. (2005) also indicated that left frontal asymmetry may be associated with heightened anxiety in children with autism. Furthermore, contrary to expectations based on previous work (Volkmar et al. 1989) the left and right frontal EEG groups did not differ on IQ suggesting that cortical processes associated with approach or avoidance behaviors may distinguish subgroups of children with autism based solely on phenotypic expression and regardless of differences in IQ.

A logical extension of this interpretation is that resting anterior EEG asymmetry may be expected to correlate with other aspects of phenotypic variability previously related to differences in approach and avoidance; such as the earlier or later onset or course of symptom presentation in children with autism (Garon et al. 2009). To examine this hypothesis 
and gather more detailed data on individual differences in developmental course and social-emotional functioning, a second study was conducted by our research group. This study was motivated by several research goals. The first goal was to replicate the observation that left frontal EEG asymmetry is associated with attenuated social symptom intensity within a larger sample of higher functioning children with autism. The sample of HFA children in this study $(N=35)$ was substantially larger than in Sutton et al. (2005, $N=23$ ). This afforded an opportunity to examine how frontal EEG asymmetry may interact with other potential modifiers of autism, such as IQ. Examining the effects of IQ was important for two reasons. Prior research suggested that active-approach versus inhibition- withdrawal may be related to higher and lower IQ respectively (Volkmar et al. 1989). Although Sutton et al. (2005) did not observe an effect of IQ on frontal asymmetry in HFA children, it was important to re-examine this issue because of the possibility that limited statistical power limited the ability to detect associations. Furthermore, our work examining other modifier processes, such as response monitoring, has indicated that modifier effects may be conditional on higher and lower IQ in research with high functioning children with autism (Henderson et al. 2006).

The second goal of this study was to determine if domains of behavior other than social symptoms were meaningfully related to anterior EEG asymmetry differences in autism. In this regard we tested two hypotheses. One was that based on the findings of Sutton et al. (2005) in which greater left frontal asymmetry was associated with anxiety symptoms in children with autism, we examined associations with a specific manifestation of anxiety, obsessive compulsive symptoms, given the common association between OCD symptoms and the broad autism phenotype (Zandt et al. 2007). We also extended the hypotheses regarding social/ emotional functioning to examine associations between resting frontal asymmetry and anger expression, given the conceptualization of anger as a negative approach-related emotion (e.g., Harmon-Jones and Allen 1998). This lead to the divergent hypothesis that left frontal asymmetry in children with autism would not only attenuate social impairments but also increase comorbid emotional problems including OCD symptoms and expressed anger.

Finally, this study was designed to address the hypothesis that anterior EEG asymmetry measures may be related to differences in the developmental course of symptom presentation in higher functioning children with autism (Mundy et al. 2007). To provide one test of this hypothesis we examined the prediction that retrospective parent report of age of onset of symptoms on the Autism Diagnostic Inventory (ADI) would be associated with anterior EEG asymmetry in higher functioning children and adolescents with autism. The rationale for this hypothesis was based on observations that individual differences in anterior EEG asymmetry measures of brain processes that are associated with approach and avoidance develop in the first 2 years of life (Davidson and Fox 1989; Field and Diego 2008; Schmidt 2008), and are moderately stable across infancy and childhood (Field and Diego 2008; Vuga et al. 2008), as well as the finding that differences in approach tendencies are associated with differences in age of appearance of clear symptoms in young children with autism (Garon et al. 2009).

\section{Methods}

\section{Participants}

The sample was comprised of 35 children (32 boys) with a prior diagnosis of Autism Spectrum Disorder without mental retardation or Higher Functioning Autism (HFA, IQs above 70). Participants were recruited from a registry at the University of Miami Center for Autism and Related Disabilities (CARD). A comparison sample included 28 children (25 boys) without ASD or mental retardation recruited from local public school regular education classrooms. All participants were right-handed and had no history of seizures. The HFA and comparison groups were matched on age, gender, and verbal and perceptual IQ as assessed on the Weschler Intelligence Scale for ChildrenFourth Edition (see Table 1). Diagnostic verification was conducted using total symptom scores on the Autism Spectrum Screening Questionnaire (ASSQ; Ehlers et al. 1999) and the Social Communication Questionnaire (SCQ; Rutter et al. 2003). The SCQ also provided subscale measures of problems specific to Social Interactions, Communication, and Restricted Interests and Repetitive Behaviors. Higher functioning children with previous community diagnosis of an autism spectrum disorder were retained in the HFA group if their parents rated them at or above established diagnostic cutoff scores of 13 for the SCQ and/ or 15 for the ASSQ (see Corsello et al. 2007; Ehlers et al. 1999 for relevant ROC data). Twenty-eight of the 35 HFA participants $(80 \%)$ met criteria on both measures. Six met or exceeded the cutoff of 15 for ASD on the ASSQ only, which exceeds the new screening criterion score of 14 recommended in a recent report of research on 9,430 children (Posserud et al. 2006). One met criteria on the SCQ but not the ASSQ. All participants in the comparison sample scored below diagnostic cutoffs on both measures.

\section{Procedure}

Each participant made two visits to the laboratory. During the first visit assessments of IQ, symptoms, and social/ 
Table 1 Descriptive data comparing diagnostic groups on age, IQ, autism symptoms, and frontal EEG asymmetry

\begin{tabular}{|c|c|c|c|c|c|c|c|}
\hline \multirow[t]{2}{*}{ Measure } & \multicolumn{3}{|c|}{ HFA group $(N=35)$} & \multicolumn{3}{|c|}{ Comparison group $(N=28)$} & \multirow[t]{2}{*}{$t$-value, $p$-value } \\
\hline & $M$ & SD & Range & $M$ & SD & Range & \\
\hline Age (years, months) & 12,2 & 2,0 & $8,1-15,2$ & 12,0 & 1,9 & $8,0-15,2$ & $.32, n s$ \\
\hline Prorated VCI & 105.00 & 15.51 & $81-140$ & 106.82 & 11.15 & $89-130$ & $-.52, n s$ \\
\hline Prorated PRI & 102.74 & 17.15 & $67-137$ & 101.75 & 7.93 & $86-117$ & $.30, n s$ \\
\hline SCQ total & 17.42 & 5.42 & $4-28$ & 3.42 & 2.57 & $0-10$ & $13.49,<.001$ \\
\hline ASSQ & 27.31 & 5.46 & $11-43$ & 5.46 & 4.53 & $0-14$ & $13.55,<.001$ \\
\hline Midfrontal asymmetry & .016 & 0.09 & $-.16-.29$ & -0.007 & 0.11 & $-.25-.18$ & $.92, n s$ \\
\hline Lateral frontal asymmetry & .017 & 0.17 & $-.34-.35$ & .022 & 0.18 & $-.23-.47$ & $-.12, n s$ \\
\hline \multicolumn{8}{|c|}{ Mid-frontal asymmetry groupings } \\
\hline Left $n$ & 19 & & & 13 & & & \\
\hline Right $n$ & 16 & & & 15 & & & \\
\hline \multicolumn{8}{|c|}{ Lateral frontal asymmetry groupings } \\
\hline Left $n$ & 18 & & & 13 & & & \\
\hline Right $n$ & 17 & & & 15 & & & \\
\hline
\end{tabular}

VCI WISC-IV verbal comprehension index, PRI WISC-IV perceptual reasoning index, SCQ total social communication questionnaire, $A S S Q$ total autism spectrum screening questionnaire

emotional functioning were conducted. During the second visit, participants completed an EEG assessment, in which a total of 6 min of resting EEG data were collected.

\section{Autism Diagnostic Interview-Revised}

Two items from the Autism Diagnostic Interview-Revised (ADI-R; Rutter et al. 2003) were used to provide retrospective data on the early course of development and symptom presentation in the HFA sample. Items of interest were: Age of first concern in months (continuous) and Age of onset-hindsight (categorically coded 0-6).

\section{IQ Assessment}

All participants were administered four subtests from The Wechsler Intelligence Scale for Children-Fourth Edition (WISC-IV, Wechsler 2003): Similarities, Vocabulary, Block Design, and Matrix Reasoning. A prorated Verbal Comprehension Index (VCI) score was computed based on the Vocabulary and Similarities subtests and a prorated Perceptual Reasoning Index (PRI) score was computed based on the Block Design and Matrix Reasoning subtests. For the purposes of data analysis, children were divided into lower versus higher IQ groups based on a median split on the VCI.

\section{Obsessions and Compulsions}

Children completed the Leyton Child Obsessional Inventory (Bamber et al. 2002; Berg et al. 1988), a 20-item selfreport measure of obsessions and compulsions designed to screen adolescents for Obsessive Compulsive Disorder (OCD). Each item was rated on a 4-point severity scale. Sample items include: "Do you go over things a lot that you have done because you aren't sure they were the right things to do?" and "Do you worry a lot if you've done something not exactly the way you like?" The scale has sound psychometric properties with a reported ICC of .90 . Of interest in the current study was the total score on the Leyton.

\section{Anger Expression}

Children provided self reports on the Pediatric Anger Expression Scale-Third Edition (PAES-III; Jacobs et al. 1989; Jacobs and Kronaizl 1991), a 15-item measure of anger expression styles. Of interest were dimensions assessing the tendency to (a) turn anger inward and (b) express anger outwardly. In previous studies (Hagglund et al. 1994), the PAES-III dimensions demonstrated adequate internal consistencies (Cronbach's alphas ranging from .54 to .72). The PAES-III has strong concurrent validity with other measures of anger expression (Hagglund et al. 1994).

\section{Resting Anterior EEG Asymmetry}

EEG was collected in a private and dimly lit testing room following the methods described in Sutton et al. (2005). Two research assistants who had previously interacted with the participant were in the room during scalp preparation and impedance checks and the participant was given the option to watch an age-appropriate video of their choice 
during the preparation period. During data collection, one research assistant stayed in the room to guide the participant through the collection protocol. Immediately prior to each participant visit, a $50 \mu \mathrm{V}, 10 \mathrm{~Hz}$ calibration signal was input into each of the channels. EEG was collected using a Lycra stretch electro-cap (Electro-Cap International Inc., Eaton, $\mathrm{OH}$ ), with electrodes embedded in positions corresponding to the 10-20 electrode system. EEG was recorded from the following sites: F7, F3, Fz, F4, F8, and $\mathrm{FCz}$ [frontal], C3, Cz, C4 [central], T7, T8 [anterior temporal], P3, Pz, P4 [parietal], O1, O2 [occipital]), and A2 (right ear lobe) with all sites referenced to the left ear lobe (A1) and a ground electrode at site AFz. EOG was recorded at the outer canthus of each eye in addition to the supraand sub-orbit of one eye. Following gentle abrasion, impedances were measured at each site and considered acceptable if each site was at or below $5 \mathrm{k} \Omega$ and each pair of homologous sites was within $2 \mathrm{k} \Omega$. Data were collected using an optically isolated, battery-powered bio-amplifier (SA Instrumentation, San Diego, CA) with a low-pass filter set at $100 \mathrm{~Hz}$ and a high-pass filter set at $0.1 \mathrm{~Hz}$. EEG signals were amplified 5,000 times and EOG signals were amplified 2,500 times. Signals were digitized at $512 \mathrm{~Hz}$ using Snap-Master acquisition software (HEM Data Corporation, Southfield, MI). During data acquisition children were instructed to minimize head movements during a sequence of twelve 30-s trials in which they alternated between having their eyes closed and having their eyes open (fixating on a blank wall four feet in front of the data acquisition chair). The order of trials (i.e., EC first or EO first) was counterbalanced across participants.

Following data collection, the EEG data were reduced and analyzed offline using software from the James Long Company. EEG signals were re-referenced to an averagedears reference configuration. Portions of each 30-s trial containing eye movement, excessive muscle movement, or other sources of artifact at any site were manually removed prior to further analysis. The re-referenced artifact-free EEG data were included in a spectral analysis using a 1-s Hanning window with adjacent windows overlapped $50 \%$. The mean total number of epochs extracted per participant was $441(\mathrm{SD}=111)$ and ranged from 199 to 680 . The mean number of epochs extracted in EO condition was 215 $(\mathrm{SD}=69)$ and in the EC condition was $244(\mathrm{SD}=77)$. For each electrode site and each of the 12 trials, alpha power was computed as the natural logarithm of power in the $8-13 \mathrm{~Hz}$ band. Analyses focused on the alpha band because power in the alpha band is more strongly related to behavioral measures of interest than is power in other bands (Davidson et al. 2000).

For each trial, asymmetry scores were calculated for homologous electrode pairs (F4/F3; F8/F7; C3/C3; P3/P4) by subtracting the log-transformed power density value of the left electrode from the paired right side electrode (e.g., ln F4-ln F3). Internal consistency reliability of the asymmetry scores were examined across all 12 trials within each region (mid-frontal, lateral frontal, central, and parietal) and were very good to excellent, averaging .87 and ranging from .82 for lateral frontal asymmetry to .90 for parietal asymmetry. Reliabilities were slightly lower, but still very good, for asymmetry scores within the EO and EC conditions separately: the range in the EO condition was .72 for lateral frontal asymmetry to .82 for central asymmetry, average .78; and in the EC condition the range was .70 for lateral frontal asymmetry to .93 for parietal asymmetry, average .79. Final alpha power estimates for each site and asymmetry scores for homologous sites were computed based on weighted averages (by number of artifact-free epochs per trial) across (a) the six EO trials, (b) the six EC trials, and (c) all twelve trials (see Sutton et al. 2005). Asymmetry scores across the EO and EC conditions were highly correlated: .75 (midfrontal), .67 (lateral frontal), .68 (central), and .58 (parietal), all $p<.001$. For the purposes of group comparisons, participants were divided into left versus right mid-frontal and lateral frontal asymmetry groups based on median splits on each measure. Given the magnitudes of the correlations across the EO and EC conditions and the excellent internal consistencies across all twelve trials, the combined asymmetry index was the primary DV of interest. However, all analyses were also conducted for the EC and EC data separately. For the sake of clarity and concision, results from this 2 nd set of analyses are only reported when they differed from those for the combined EO \& EC index. Divergent results were limited to a few analyses of the EC alone data.

\section{Results}

Preliminary correlation analyses revealed no significant relations between verbal IQ and any measures of alpha band power or EEG asymmetry across the entire sample or within the HFA or comparison group alone. Boys and girls did not differ on verbal IQ or any measures of alpha band power or EEG asymmetry. The relations between diagnostic group and alpha-band cortical power were examined using a 4 (region: midfrontal, lateral frontal, central, parietal) $\times 2$ (hemisphere: left, right) $\times 2$ (diagnostic group: HFA, comparison) repeated measures ANOVA. Regional differences in alpha power were qualified by interactions between region and (a) diagnostic group, $F(3,59)=5.97$, $p=.001, \eta_{\mathrm{p}}^{2}=.23$, and (b) hemisphere, $F(3,59)=3.90$, $p=.01, \eta_{\mathrm{p}}^{2}=.16$. Across all participants, power was greatest in the parietal, followed by central, mid-frontal, and lateral frontal regions. Within each diagnostic group, all paired comparisons between regions were highly 
significant (all $p<.001$ ). Diagnostic groups had comparable alpha power levels across regions with the exception of the central region where participants in the HFA group tended to have less alpha power compared to participants in the comparison group, $t(61)=-1.81, p=.07$. As such, the interaction between region and diagnostic group appeared to be driven by the relatively smaller (although still highly significant) difference in power between central and lateral frontal regions for the HFA group relative to the comparison group.

To interpret the region by hemisphere interaction, paired comparisons of alpha power were conducted across homologous sites. Across the entire sample, mean alpha power estimates did not differ by hemisphere at midfrontal, lateral frontal, or central sites. However, there was greater right parietal (p4) power compared to left parietal (p3) power, $t(62)=-2.75, p=.008$. The lack of a 3-way interaction between diagnostic group, region and hemisphere suggested that diagnostic groups did not differ on mean levels of asymmetry at any region. This was confirmed with a MANOVA examining diagnostic group differences on the computed asymmetry scores which showed no effect of diagnostic group, $F(4,58)=.53$, $n s$. Consistent with this finding, there were no differences in the frequencies of HFA and comparison children assigned to Left versus Right frontal asymmetry groups on the basis of median splits of either mid-frontal, $\chi^{2}(1)=.38, n s$, or lateral frontal, $\chi^{2}(1)=.07, n s$ asymmetry scores (see Table 1). Mean power values and asymmetry scores are presented separately by diagnostic group in Table 2 .

\section{Frontal Asymmetry and IQ in Relation to Autism} Symptom Severity

To examine the relations between frontal EEG asymmetry, IQ and differences in symptom severity a 2 (Diagnostic Group: HFA, comparison) $\times 2$ (VIQ Group: relatively low, relatively high) $\times 2$ (Asymmetry Group: Left or Right) MANOVA was computed with ASSQ total score, SCQ Social Interaction, SCQ Communication, and SCQ Repetitive Behaviors as the dependent variables. Separate MANOVAs were conducted using lateral frontal and mid frontal asymmetry groupings.

Variations in symptom severity were associated with a significant three-way interaction between Diagnostic Group, IQ, and lateral frontal asymmetry, $F(4,51)=5.70$, $p=.001, \eta_{\mathrm{p}}^{2}=.31$. Post-hoc univariate analyses revealed that using the combined asymmetry index, the interaction was significant for the SCQ communication domain, $F(1$, $55)=4.17, \quad p=.04, \quad \eta_{\mathrm{p}}^{2}=.07$, and the interactions approached significance for both the SCQ social and SCQ repetitive behavior domains, $F(1,55)=3.27, p=.07$, $\eta_{\mathrm{p}}^{2}=.06, \quad$ and $\quad F(1, \quad 55)=2.88, \quad p=.09, \quad \eta_{\mathrm{p}}^{2}=.05$,
Table 2 EEG alpha-power (ln) and asymmetry measures by diagnostic group

\begin{tabular}{|c|c|c|c|c|}
\hline \multirow[t]{2}{*}{ Measure } & \multicolumn{2}{|c|}{ HFA group } & \multicolumn{2}{|c|}{ Comparison group } \\
\hline & $M$ & SD & $M$ & SD \\
\hline \multicolumn{5}{|l|}{ Alpha-band power } \\
\hline Left mid-frontal (F3) & 3.30 & .74 & 3.52 & .54 \\
\hline Right mid-frontal (F4) & 3.31 & .73 & 3.51 & .51 \\
\hline Left lateral frontal (F7) & 2.76 & .67 & 2.85 & .52 \\
\hline Right lateral frontal (F8) & 2.77 & .69 & 2.87 & .51 \\
\hline Left central (C3) & 3.56 & .74 & 3.90 & $.62^{\mathrm{t}}$ \\
\hline Right central (C4) & 3.56 & .77 & 3.87 & $.62^{\mathrm{t}}$ \\
\hline Left parietal (P3) & 4.02 & .85 & 4.32 & .70 \\
\hline Right parietal (P4) & 4.09 & .89 & 4.38 & .72 \\
\hline \multicolumn{5}{|l|}{ Asymmetry } \\
\hline Mid-frontal & .016 & .09 & -.007 & .11 \\
\hline Lateral frontal & .017 & .17 & .022 & .18 \\
\hline Central & .003 & .16 & -.028 & .16 \\
\hline Parietal & .072 & .20 & .063 & .19 \\
\hline
\end{tabular}

${ }^{\mathrm{t}}$ Diagnostic group difference $p<.10$

respectively. Within the HFA sample, children with left lateral frontal asymmetry and lower IQs had lower mean SCQ Social Interaction symptom scores compared to both lower IQ HFA children with right lateral frontal asymmetry, $t(16)=-3.12, p=.007$, and higher IQ HFA children with left lateral frontal asymmetry, $t(16)=-1.84, p=$ .08. In contrast, lateral frontal asymmetry was not associated with differences in social symptoms between higher IQ HFA children, $t(15)=.27, n s$, and neither lateral frontal asymmetry or IQ were related to social symptoms in the comparison sample (see Table 3). On the SCQ Communication domain, IQ and lateral frontal asymmetry were not related to individual differences in the HFA sample, however; within the comparison sample, lower IQ children with left lateral frontal asymmetry had the lowest levels of communication symptoms, significantly less than higher IQ children with left lateral frontal asymmetry, $t(11)=-$ $2.83, p=.016$ and approaching significance compared to lower IQ children with right frontal asymmetry, $t(14)=$ $-1.86, p=.08$ (see Table 3). On the SCQ repetitive behavior domain, HFA children were consistently higher than children in the comparison sample, except in the case of higher IQ children with left frontal asymmetry, where the diagnostic groups did not differ significantly, $t(10)=$ 1.30, ns (see Table 3).

Using the EC index alone, the 3-way interaction between Diagnostic Group, IQ, and lateral frontal asymmetry was significant, $F(4,51)=2.89, p=.03, \eta_{\mathrm{p}}^{2}=.19$. However, post-hoc univariate analyses revealed that this was driven exclusively by a trend for an effect for the SCQ communication domain scores, $F(1,54)=3.62, p=.06$, 
Table 3 Diagnostic, age and frontal asymmetry group differences on the diagnostic measures

\begin{tabular}{|c|c|c|c|c|c|c|c|c|}
\hline & \multicolumn{4}{|c|}{ HFA group $N=35$} & \multicolumn{4}{|c|}{ Comparison group $N=28$} \\
\hline & \multicolumn{2}{|l|}{ LFA $(n=18)$} & \multicolumn{2}{|l|}{ RFA $(n=17)$} & \multicolumn{2}{|c|}{$\operatorname{LFA}(n=13)$} & \multicolumn{2}{|c|}{ RFA $(n=15)$} \\
\hline & $\begin{array}{l}\text { Lower IQ } \\
(n=11)\end{array}$ & $\begin{array}{l}\text { Higher IQ } \\
(n=7)\end{array}$ & $\begin{array}{l}\text { Lower IQ } \\
(n=7)\end{array}$ & $\begin{array}{l}\text { Higher IQ } \\
(n=10)\end{array}$ & $\begin{array}{l}\text { Lower IQ } \\
(n=8)\end{array}$ & $\begin{array}{l}\text { Higher IQ } \\
(n=5)\end{array}$ & $\begin{array}{l}\text { Lower IQ } \\
(n=8)\end{array}$ & $\begin{array}{l}\text { Higher IQ } \\
(n=7)\end{array}$ \\
\hline \multicolumn{9}{|l|}{ Lateral frontal asymmetry $^{1}$} \\
\hline ASSQ & $25.09 \mathrm{a}(8.59)$ & $27.00_{\mathrm{a}}(7.14)$ & $25.57_{\mathrm{a}}(7.73)$ & $31.20_{\mathrm{a}}(8.04)$ & $5.37_{\mathrm{b}}(5.01)$ & $7.80_{\mathrm{b}}(6.26)$ & $5.37_{\mathrm{b}}(4.10)$ & $4.00_{\mathrm{b}}(2.89)$ \\
\hline SCQ social interaction ${ }^{3}$ & $4.45_{\mathrm{a}}(1.21)$ & $6.43_{\mathrm{b}}(3.26)$ & $8.14_{\mathrm{b}}(3.54)$ & $6.00_{\mathrm{ab}}(3.23)$ & $1.12_{\mathrm{c}}(0.99)$ & $1.60_{c}(0.55)$ & $0.62_{\mathrm{c}}(0.74)$ & $1.14_{c}(1.07)$ \\
\hline SCQ communication ${ }^{2}$ & $6.36_{\mathrm{a}}(1.63)$ & $6.14_{\mathrm{a}}(1.07)$ & $5.14_{\mathrm{a}}(2.54)$ & $6.60_{\mathrm{a}}(2.06)$ & $0.87_{\mathrm{b}}(0.64)$ & $2.80_{\mathrm{c}}(1.79)$ & $1.75_{\mathrm{c}}(1.16)$ & $1.86_{\mathrm{bc}}(1.57)$ \\
\hline SCQ repetitive behavior ${ }^{3}$ & $3.90_{\mathrm{a}}(2.02)$ & $3.57_{\mathrm{ac}}(2.22)$ & $3.57 \mathrm{a}(1.51)$ & $4.80_{\mathrm{a}}(1.93)$ & $0.37_{\mathrm{b}}(0.74)$ & $1.80_{\mathrm{bc}}(2.49)$ & $0.38_{\mathrm{b}}(0.74)$ & $0.43_{\mathrm{b}}(0.79)$ \\
\hline \multicolumn{9}{|l|}{ Mid-frontal asymmetry ${ }^{1}$} \\
\hline ASSQ & $26.10_{\mathrm{a}}(8.10)$ & $27.55_{\mathrm{a}}(7.07)$ & $24.25_{\mathrm{a}}(8.46)$ & $31.62_{\mathrm{a}}(8.36)$ & $4.75_{b}(4.65)$ & $6.00_{\mathrm{b}}(6.04)$ & $6.00_{b}(4.41)$ & $5.25_{\mathrm{b}}(4.35)$ \\
\hline SCQ social interaction & $5.20_{\mathrm{a}}(2.20)$ & $6.56_{\mathrm{a}}(2.83)$ & $6.75_{\mathrm{a}}(3.77)$ & $5.75_{\mathrm{a}}(6.25)$ & $1.25_{\mathrm{b}}(0.89)$ & $1.00_{\mathrm{b}}(1.00)$ & $0.50_{\mathrm{b}}(0.76)$ & $1.57_{\mathrm{b}}(0.79)$ \\
\hline $\begin{array}{l}\text { SCQ social } \\
\text { communication }\end{array}$ & $6.20_{\mathrm{a}}(1.99)$ & $6.22_{\mathrm{a}}(0.97)$ & $5.50_{\mathrm{a}}(2.20)$ & $6.62_{\mathrm{a}}(2.33)$ & $0.75_{\mathrm{b}}(0.71)$ & $2.60_{\mathrm{b}}(1.81)$ & $1.87_{\mathrm{b}}(0.99)$ & $2.00_{\mathrm{b}}(1.63)$ \\
\hline $\begin{array}{c}\text { SCQ repetitive } \\
\text { behaviors }^{2}\end{array}$ & $4.70_{\mathrm{a}}(1.64)$ & $3.56_{\mathrm{abd}}(1.94)$ & $2.62_{b}(1.30)$ & $5.12_{\mathrm{a}}(2.03)$ & $0.37_{\mathrm{c}}(0.74)$ & $1.60_{\mathrm{cd}}(2.61)$ & $0.37_{c}(0.72)$ & $0.57_{\mathrm{c}}(0.79)$ \\
\hline
\end{tabular}

Means within a row with different subscripts differ significantly

$S C Q$ social communication questionnaire, $A S S Q$ autism spectrum screening questionnaire

${ }^{1}$ Significant multivariate diagnostic by frontal by IQ group effect, $p<.001$

${ }^{2}$ Significant univariate diagnostic by frontal by IQ group effect, $p<.05$

${ }^{3}$ Trend univariate diagnostic by frontal by IQ group effect, $p<.10$

$\eta_{\mathrm{p}}^{2}=.06$. Similar to the observations for the combined EO-EC data, children in the comparison sample with relatively lower IQ and left lateral frontal asymmetry had the fewest communicative impairments relative to both higher IQ children with left lateral frontal asymmetry, $t(11)=$ $-2.84, p=.016$ and lower IQ children with right frontal asymmetry, $t(13)=-2.52, p=.03$.

A comparable three way interaction (diagnostic group $\times$ IQ $\times$ mid-frontal asymmetry) was significant when mid-frontal asymmetry group was used as an independent variable, $F(4,52)=5.57, p=.001, \eta_{\mathrm{p}}^{2}=.30$; however the only significant univariate effect was for the SCQ restricted/repetitive behavior domain, $F(1,55)=$ $8.52, p=.005, \eta_{\mathrm{p}}^{2}=.13$. As was the case using lateral frontal asymmetry, post hoc comparisons revealed that HFA children were consistently higher in restricted/repetitive behaviors except when comparing higher IQ children with left midfrontal asymmetry, where diagnostic group was unrelated to ratings on this domain, $t(12)=1.6$, ns. Within the HFA group, lower IQ children with right midfrontal asymmetry had the lowest levels of restrictive/ repetitive behavior, significantly less than children in both the lower IQ/left frontal group, $t(16)=2.92, p=.01$ and children in the higher $\mathrm{IQ} /$ right frontal group, $t(14)=-2.93$, $p=.01$ (see Table 3).

Comparable relations were found using the EC index alone, however post hoc analyses revealed that HFA participants were consistently rated higher than comparison participants in restricted/repetitive behaviors, even when looking at the higher IQ children with left mid-frontal asymmetry. Thus, the interaction was driven by the relations within the HFA group where, as was the case using the combined asymmetry index, lower IQ children with right mid-frontal EC asymmetry had the lower levels of restrictive/repetitive behavior, compared to HFA children in the lower IQ/left frontal group, $t(16)=2.92, p=.01$ and HFA children in the higher IQ/right frontal group, $t(14)=2.93, p=.01$.

Frontal Asymmetry and IQ in Relation to Emotional Functioning

The effects of Diagnostic Group, frontal EEG asymmetry, and IQ on self-reported (1) Obsessions and Compulsions and (2) Anger Expression were examined in separate analyses. Mean scores on the total obsessions and compulsions score on the Leyton were associated with IQ, $F(1$, $55)=6.10, p=.017, \eta_{\mathrm{p}}^{2}=.10$. Regardless of diagnostic group, children with lower verbal IQs reported more obsessions and compulsions. In addition, there was a main effect of diagnostic group on the Leyton self-report total score; however, this effect was qualified by an interaction with lateral frontal asymmetry, $F(1,55)=9.12, p=.004$, $\eta_{\mathrm{p}}^{2}=0.14$. Post hoc comparisons revealed that children in 
the HFA group with left lateral frontal asymmetry reported significantly more obsessive and compulsive thoughts and behaviors relative to children in the HFA group with right frontal asymmetry, $t(33)=3.71, p=.001$ and relative to children in the comparison sample with left lateral frontal asymmetry, $t(29)=3.84, p=.001$. In contrast, children in the HFA and comparison groups who had right lateral frontal asymmetry did not differ in self-reported obsessions and compulsions, $t(30)=-.78, n s$.

Identical results were obtained using mid-frontal asymmetry groups as the independent variable. Again, Leyton total scores were predicted by the interaction of diagnostic and mid-frontal asymmetry groups, $F(1,55)=$ 5.63, $p=.02, \eta_{\mathrm{p}}^{2}=.09$. Post hoc comparisons revealed that children in the HFA group with left mid-frontal asymmetry reported significantly more obsessions and compulsions compared to HFA children with right midfrontal asymmetry, $t(33)=2.19, p=.036$, as well as comparison children with left mid-frontal asymmetry, $t(30)=3.16, p=.004$. Within the comparison group, mid-frontal asymmetry was not associated with differences on the Leyton, $t(26)=-1.08, n s$, (see Fig. 2).

Lateral asymmetry was unrelated to patterns of anger expression either alone or in combination with IQ and/or diagnostic group. However, a 2 (diagnostic group) $\times 2$ (mid-frontal asymmetry group) $\times 2$ (IQ group) MANOVA with anger-inward and anger-outward as the dependent variables revealed a significant interaction between diagnostic group and mid-frontal asymmetry, $F(2,54)=3.60$, $p=.03, \eta_{\mathrm{p}}^{2}=.12$. Relative to HFA participants with right

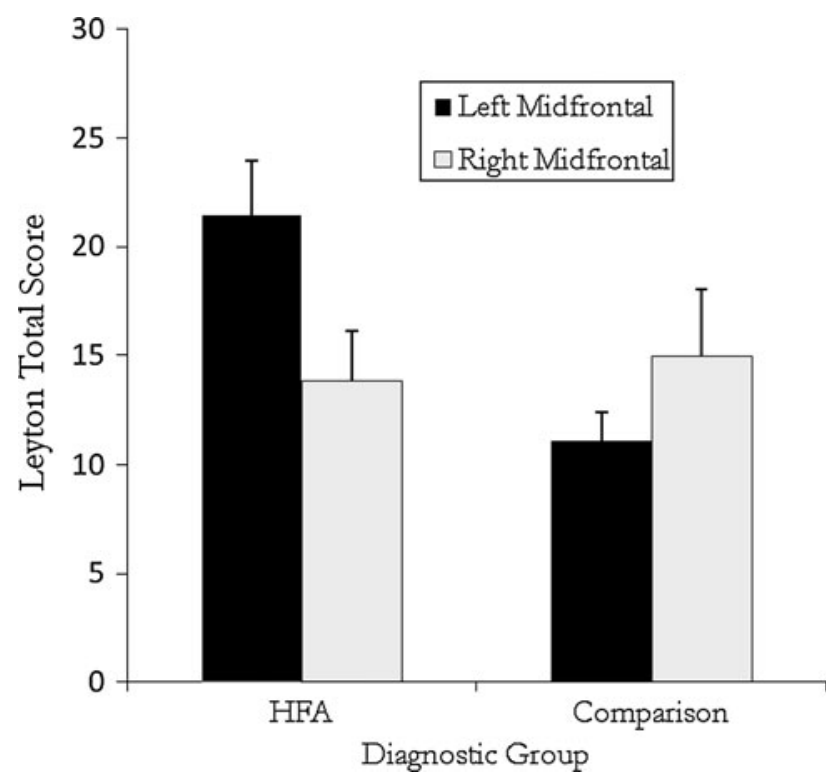

Fig. 2 Leyton total score as a function of diagnostic group and midfrontal EEG asymmetry mid-frontal asymmetry, HFA participants with left midfrontal asymmetry reported higher levels of outwardly expressed anger, $t(33)=3.10, p=.004$ and tended to report lower levels of inwardly directed anger, $t(33)=-$ $1.77, p=.08$. The HFA participants with left mid-frontal asymmetry also reported significantly higher levels of outwardly directed anger compared to comparison participants with left mid-frontal asymmetry, $t(30)=2.08$, $p=.04$. Within the comparison sample, mid-frontal asymmetry was not associated with a significant difference in anger expression (see Fig. 3a, b). Comparable associations were found using the EC asymmetry index, however, the interaction between diagnostic group and mid-frontal asymmetry was reduced to a trend, $F(2,53)=3.02$, $p=.057, \eta_{\mathrm{p}}^{2}=.10$. Post hoc analyses revealed comparable mean differences among the groups as those reported above based on the combined mid-frontal asymmetry index.

Frontal Asymmetry and IQ in Relation to Age of First Concern

For children in the HFA sample, parents completed ADI items relating to the age at which they first had concerns about their child's development. A 2 (Lateral Asymmetry Group) $\times 2$ (IQ Group) ANOVA with ADI parent retrospective report of Age of First Concern (in months) revealed a significant effect of lateral frontal asymmetry, $F(1,31)=5.41, p=.027, \eta_{\mathrm{p}}^{2}=.15$. On average, parents of HFA children with left lateral frontal asymmetry reported an average age of first concern of 40.39 months $(\mathrm{SD}=24.01)$ whereas parents of HFA children with right lateral frontal asymmetry reported having concerns much earlier at an average age of only 22.29 months $(\mathrm{SD}=19.92)$. Similarly, there was a significant association between lateral frontal asymmetry group and categorical reports of age of first concern in hindsight, $\chi^{2}$ (6) $=13.18, p=.04$. Parents reported that they were first concerned at the earliest rated point in development (prior to 12 months of age) for 10 of 17 HFA children with right lateral frontal asymmetry. In contrast, only 1 of 18 HFA children with left lateral frontal asymmetry received this rating (see Fig. 4). Patterns were comparable, but not statistically significant, when looking at mid-frontal asymmetry groups.

Post hoc Analyses Limiting Sample to Male Participants

Consistent with gender differences in the prevalence of autism, our sample was largely made-up of male participants. While there were no gender differences on any of the predictor variables (age, EEG asymmetry), we conducted 
Fig. 3 a PAES-III anger in scores as a function of diagnostic group and midfrontal EEG asymmetry. b PAES-III anger out scores as a function of diagnostic group and mid-frontal EEG asymmetry

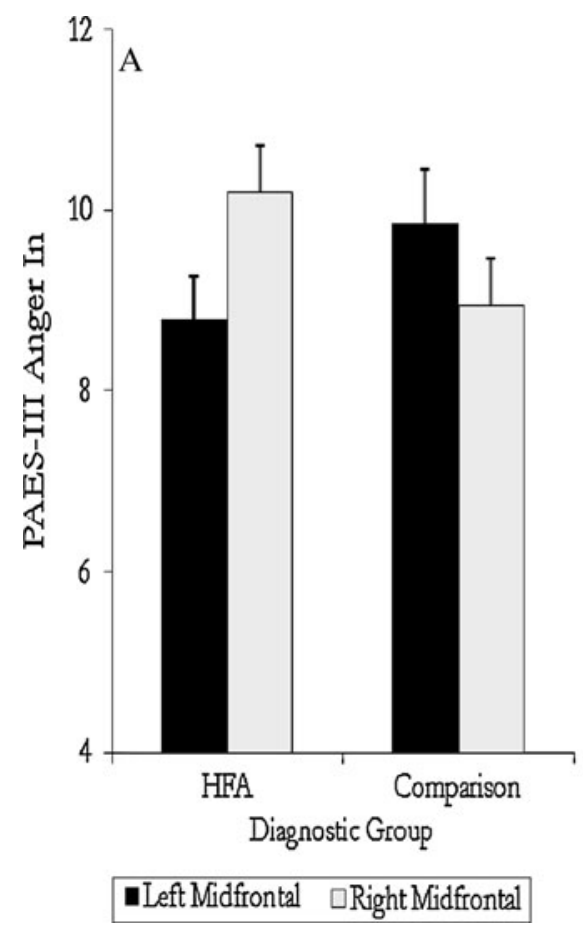

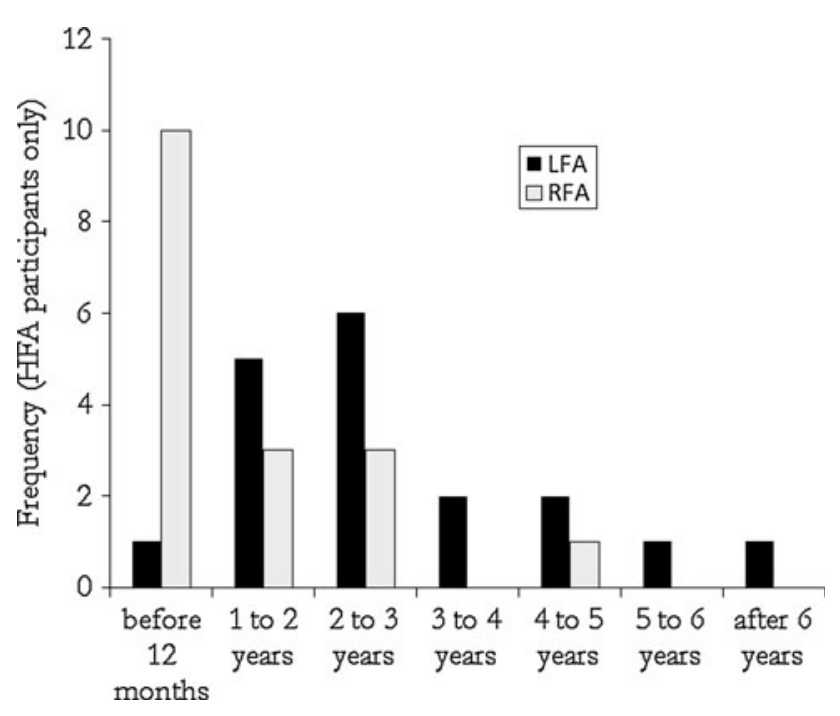

Fig. 4 Retrospective parent reports of recollection of age of onset of symptomatic behaviors for children and adolescents with left or right lateral frontal EEG asymmetry

post-hoc analyses to determine if the results held when the sample was limited to male participants. All results remained significant and of comparable magnitude with only minor exceptions when looking at symptom severity. Specifically, when limiting the sample to boys, the trend for the three way interaction between diagnostic group, lateral frontal asymmetry, and IQ in the prediction of restricted/repetitive behaviors was reduced to non-significance, $F(1,49)=1.40, p=.24, \eta_{\mathrm{p}}^{2}=.03$. When looking at mid-frontal asymmetry in relation to symptom severity, the significant interaction between diagnostic group, midfrontal asymmetry, and IQ in predicting symptom severity was extended to the SCQ Communication domain, $F(1$, $49)=4.24, p=.04, \eta_{\mathrm{p}}^{2}=.08$. Post hoc comparisons revealed a pattern very similar to that observed in the whole sample in the prediction of communication symptoms. IQ and mid-frontal asymmetry were not related to SCQ communication scores in the HFA sample, but in the comparison sample, participants with lower IQ and left mid-frontal asymmetry had significantly lower scores compared to both comparison participants with lower IQ and right mid-frontal asymmetry, $t(11)=-3.71, p=.003$ and comparison participants with higher IQ and left frontal asymmetry, $t(11)=-2.63, p=.02$.

\section{Discussion}

For 30 years it has been observed that differences in approach and avoidance tendencies form a major axis of variability in autism (Wing and Gould 1979). Consistent with this observation the results of the current study, along with those of Sutton et al. (2005) that frontally mediated individual differences in brain processes associated with behavioral approach and avoidance tendencies may contribute to significant variability in symptom presentation and patterns of behavior and ideation related to anger and anxiety. Furthermore, the source of differenced in these 
brain processes appeared to be related to generic rather than syndrome specific factors because there was little evidence of diagnostic group differences in cortical alphaband power or frontal EEG asymmetry in the current study.

Patterns of right resting frontal EEG asymmetry are thought to reflect relatively greater activation of a neural network including the frontal cortex, amygdala, septohippocampal system, and brainstem that regulates responses to signals of punishment, non-reward, and novelty. Consequently, individuals with relatively greater right anterior versus left anterior brain activity tend to exhibit inhibition of movement towards goals and withdrawal from novel situations and social interactions (e.g. Davidson 2002; Gray 1994; Sutton and Davidson 1997). Alternatively, left anterior EEG asymmetry is thought to involve a dopaminergically-mediated network of the left middlesuperior frontal and pre-central gyrus, the left inferior parietal lobe, as well as bilateral activation in the dorsolateral prefrontal cortex, orbital frontal, and anterior cingulate cortices. It functions to regulate responses to signals of reward by initiating and modifying movement towards goals. Individuals with relatively heightened left frontal activity tend to exhibit more activation of goal-directed, reward seeking behavior and to anticipate positive affective states when exposed to cues of potential reward, as well as anger/frustration when approach-related goals are blocked (Gray 1994; Harmon-Jones and Allen 1998; Pizzagalli et al. 2005; Van Honk and Schutter 2006).

The active goal directed and reward seeking behavior associated with left frontal asymmetry may contribute to patterns of behavior that are relatively inconsistent with what the prototype of autism typified by withdrawal and avoidance. Alternatively, the presentation of autism in children with a bias toward inhibition and withdrawal, associated with right frontal asymmetry, may result in a perceived accentuation of prototypical symptoms of the syndrome in older HFA children. The results of this study suggest that this effect on symptom ratings may be more pronounced in HFA children with IQs in the borderline range, and less prominent among children in the higher ranges of IQ. This observation reminds us that IQ is itself a significant modifier of the expression of autism. Understanding its role in the expression, detection, developmental course and treatment of autism remains a long standing and under-scrutinized issue in research on autism.

In contrast to social symptoms, the relations between anterior EEG asymmetry and emotional functioning and developmental history observed in the current study were not moderated by IQ. Parents recalled having concerns and noticing symptoms at a much later age for left frontal rather than right frontal children and adolescents. Again it may be that tendency toward active engagement may mask early symptoms or make the initial onset of symptoms less noticeable within this subgroup of ASD children. This notion may help to explain both the retrospective data in this study and the prospective observations of an association between approach tendencies and later apparent symptom onset in young children at risk for autism (Garon et al. 2009). It is also reasonable to expect that approach and reward seeking behavior patterns are associated with more positive, constructivist and responsive engagement with intervention activities (Koegel et al. 1995). This affords one perspective on how the brain processes associated with left anterior EEG asymmetry examined in this study may also help to explain the observation of Beglinger and Smith (2005), as well as Sherer and Schreibman (2005) that approach behaviors are positively associated with behavioral intervention response tendencies in toddler and young children with autism.

On the negative side, left frontal asymmetry in children with autism was associated with increases in self- reports of outward expressions of anger and heightened symptoms of obsessive compulsive disorder. The former observation is consistent with theory that suggests that the greater goal directed and reward seeking pattern of behavior in people with left anterior EEG asymmetry may lead them to more frustration with unmet social-communication goals and it is this frustration that contributes to their increased tendency to express anger (Harmon-Jones and Allen 1998; van Honk and Schutter 2006). Alternatively, the latter observation appears to be inconsistent with the general notion that anxiety related symptoms are more frequently exhibited by people with right frontal EEG asymmetry (Davidson 2002). However, Sutton et al. (2005) also reported that social anxiety was more prominent in higher functioning children with left rather than right frontal EEG asymmetry. One possible explanation raised in the discussion of those data was based on the proposal by Heller et al. (1997) is that symptoms of anxiety associated with anxious apprehension, such as worry, are related to left anterior cortical functioning, whereas anxious arousal including panic is associated with right cortical functioning. Subsequently, Mathersul et al. (2008) have reported empirical data that strongly supports the left frontal association with anxious apprehension and worry in a study of 428 adults. So the current working hypothesis is that, just as in typical adults, left frontal asymmetry is associated with a greater tendency towards active cognitive worry, including obsessive compulsive symptoms, among HFA children.

The common thread to each point of this discussion is that anterior EEG asymmetry measurement of a generic dimension of human individual differences may make a specific contribution to the more precise assessments of diagnostic subgroups among children with autism. Diagnostic subgroups may be best defined by process measures that are linked to clinically significant differences in 
intervention responses and/or course of development of autism, and/or intensity of symptom presentation. Theory and now two studies indicate that measures of EEG asymmetry may provide a useful measure of approach and avoidance tendencies that contribute to clinically relevant sub-grouping of children with autism. Moreover, because anterior EEG asymmetry may be measured in children at different points in development, from infancy through school age (Schmidt 2008; Vuga et al. 2008), critical hypotheses about the potential contributions of frontally mediated approach and avoidance tendencies to differences in developmental course or response to intervention are open to empirical tests through prospective longitudinal studies beginning early in life. For example, it may be revealing to include asymmetry measures in infant sibling research to determine if they help to explain the types of early differences in approach behaviors and apparent symptom onset previously observed by Garon et al. (2009). They may also be useful to include in treatment studies to determine if they help us to understand the types of differences in approach behavior and childhood intervention responsiveness observed by Beglinger and Smith (2005), as well as Sherer and Schreibman (2005). Finally, the results of this study, as well as those of the seminal study by Sutton et al. (2005), suggest that measures of EEG asymmetry may be informative in research on anxiety disorders and their treatment in autism (e.g. Wood et al. 2009).

Of course much work needs to be done before any possible clinical potential of EEG asymmetry measurement can be realized. We need a better understanding of optimal methods of EEG asymmetry measurement, as well as psychometrics and the equivalence measurement validity across children. In the current study we observed excellent internal consistency of measurement, few differences in results across a sample of boys versus boys and girls with autism, but some differences in when only analyzing eyesclosed data. The latter may be important. However, in this study it was not clear if disparities using EC measurement alone were meaningful, or the result of variance in power of analysis due to lower EC versus composite EC \& OC measure reliability.

Open Access This article is distributed under the terms of the Creative Commons Attribution Noncommercial License which permits any noncommercial use, distribution, and reproduction in any medium, provided the original author(s) and source are credited.

\section{References}

Bamber, D., Tamplin, A., Park, R. J., Kyte, Z. A., \& Goodyer, I. M. (2002). Development of a short leyton obsessional inventory for children and adolescents. Journal of American Academy of Child and Adolescent Psychiatry, 41, 1246-1252.
Beglinger, L., \& Smith, T. (2005). Concurrent validity of social subtypes and IQ after early intensive behavioral intervention in children with autism. Journal of Autism and Developmental Disorders, 35, 295-303.

Berg, C. Z., Whitaker, A., Davies, M., Falment, M. F., \& Rapoport, J. L. (1988). The survey form of the Leyton obsessional inventorychild version: Norms from an epidemiological study. Journal of the American Academy of Child and Adolescent Psychiatry, 27, 759-763.

Corsello, C., Hus, V., Pickles, A., Risi, S., Cook, E. H. Jr., Leventhal, B. L., \& Lord, C. (2007). Between a ROC and a hard place: Decision making and making decisions about using the SCQ. Journal of Child Psychology and Psychiatry, 48, 932-940.

Davidson, R. J. (2002). Anxiety and affective style: Role of the prefrontal cortex and amygdala. Biological Psychiatry, 51, 68-80.

Davidson, R. J., \& Fox, N. A. (1989). Frontal brain asymmetry predicts infants' response to maternal separation. Journal of Abnormal Psychology, 98, 127-131.

Davidson, R. J., Jackson, D. C., \& Larson, C. L. (2000). Human electroencephalography. In J. T. Cacioppo, L. G. Tassinary, \& G. G. Berntson (Eds.), Handbook of Psychophysiology (pp. 27-52). New York: Cambridge University Press.

Dawson, G., Klinger, L. G., Panagiotides, H., Lewy, A., \& Castelloe, P. (1995). Subgroups of autistic children based on social behavior display distinct patterns of brain activity. Journal of Abnormal Child Psychology, 23, 569-583.

Ehlers, S., Gillberg, C., \& Wing, L. (1999). A screening questionnaire for Asperger syndrome and other high-functioning autism spectrum disorders in school age children. Journal of Autism and Developmental Disorders, 29, 129-140.

Field, T., \& Diego, M. (2008). Maternal depression effects on infant frontal EEG asymmetry. International Journal of Neuroscience, 118, 1081-1108.

Garon, N., Bryson, S. E., Zwaigenbaum, L., Smith, I. M., Brian, J., Roberts, W., et al. (2009). Temperament and its relationship to autistic symptoms in a high-risk infant sib cohort. Journal of Abnormal Child Psychology, 37, 59-78.

Gray, J. A. (1994). Three fundamental emotion systems. In P. Ekman \& R. J. Davidson (Eds.), The nature of emotion: Fundamental questions (pp. 243-247). New York: Oxford University Press.

Hagglund, K. J., Clay, D. L., Frank, R. G., Beck, N. C., Kashani, J. H., Hewett, J., et al. (1994). Assessing anger expression in children and adolescents. Journal of Pediatric Psychology, 19, 291-304.

Harmon-Jones, E., \& Allen, J. J. B. (1998). Anger and frontal brain activity: EEG asymmetry consistent with approach motivation despite negative affective valence. Journal of Personality and Social Psychology, 74, 1310-1316.

Heller, W., Nitschke, J. B., Etienne, M. A., \& Miller, G. A. (1997). Patterns of regional brain activity differentiate types of anxiety. Journal of Abnormal Psychology, 106, 376-385.

Henderson, H. A., Schwartz, C. B., Mundy, P. C., Burnette, C. P., Sutton, S. K., Zahka, N. E., et al. (2006). Error monitoring, the anterior cingulate cortex and differences in social behavior in autism. Brain and Cognition, 61, 96-109.

Jacobs, G. A., \& Kronaizl, C. (1991). Pediatric anger in rural impoverished communities. Paper presented at the annual meeting of the American Psychological Association, San Francisco, CA.

Jacobs, G., Phelps, M., \& Rohrs, B. (1989). Assessment of anger expression in children: The pediatric anger expression scale. Personality and Individual Differences, 10, 59-65.

Koegel, R. L., Koegel, L. K., Frea, W. D., \& Smith, A. E. (1995). Emerging interventions for children with autism: Longitudinal and lifestyle implications. In R. L. Koegel \& L. K. Koegel (Eds.), Teaching children with autism strategies for initiating 
positive interactions and improving learning opportunities (pp. 1-15). Baltimore: Brookes.

Mathersul, D., Williams, L. M., Hopkinson, P. J., \& Kemp, A. H. (2008). Investigating models of affect: Relationships among EEG alpha asymmetry, depression, and anxiety. Emotion, 8, $560-572$.

Meyer, J., Mundy, P., Vaughan Van Hecke, A., \& Durocher, J. (2006). Social-attribution processes and comorbid psychiatric symptoms in Asperger syndrome. Autism, 10, 383-402.

Mundy, P., Henderson, H., Inge, A., \& Coman, D. (2007). The modifier model of autism and higher functioning children. Research and Practice for Persons with Severe Disabilities, Special Autism Issue, 32, 124-139.

O'Brien, S. (1996). The validity and reliability of the wing subgroups questionnaire. Journal of Autism and Developmental Disorders, 26, 321-335.

Pizzagalli, D. A., Sherwood, R. J., Henriques, J. B., \& Davidson, R. J. (2005). Frontal brain asymmetry and reward responsiveness: A source-localization study. Psychological Science: A journal of the American Psychological Society, 16, 805-813.

Posserud, M., Lundervold, A., \& Gillberg, C. (2006). Autism features in a total population study of 7-9 year-old children assessed with the ASSQ. Journal of Child Psychology and Psychiatry, 47, $167-175$

Rutter, M., Bailey, A., \& Lord, C. (2003a). The social communication questionnaire. Los Angeles, CA: Western Psychological Services.

Rutter, M., LeCouteur, A., \& Lord, C. (2003b). Autism diagnostic interview-revised. Los Angeles, CA: Western Psychological Services.

Schmidt, L. (2008). Resting frontal EEG asymmetry and their relation to heart rate and temperament in 9 month olds. Personality and Individual Differences, 44, 216-222.

Sherer, M., \& Schreibman, L. (2005). Individual behavioral profiles and predictors of treatment effectiveness for children with autism. Journal of Consulting and Clinical Psychology, 73, $525-538$.

Sutton, S., Burnette, C., Mundy, P., Meyer, J., Vaughan, A., Sanders, C., et al. (2005). Resting cortical brain activity, social impairments, and comorbidity in high functioning children with autism. Journal of Child Psychology and Psychiatry, 46, 211-222.

Sutton, S. K., \& Davidson, R. J. (1997). Prefrontal brain asymmetry: A biological substrate of the behavioral approach and inhibition systems. Psychological Science, 8, 204-210.

Van Honk, J., \& Schutter, D. (2006). From affective valence to motivational direction: The forntal asymmetry of emotion revised. Psychological Science, 17, 963-965.

Volkmar, F. R., Cohen, D. J., Bregman, J. D., Hooks, M. Y., \& Stevenson, J. M. (1989). An examination of social typologies in autism. Journal of the American Academy of Child and Adolescent Psychiatry, 28, 82-86.

Vuga, M., Fox, N., Cohn, J., Kovacs, M., \& George, C. (2008). Long term stability of electroencephalographic asymmetry and power in 3-9 year-old children. International Journal of Psychophysiology, 67, 70-77.

Wechsler, D. (2003). Wechsler intelligence scale for children (4th ed.). San Antonio, TX: The Psychological Corporation.

Wing, L., \& Gould, J. (1979). Severe impairments of social interaction and associated abnormalities in children: Epidemiology and classification. Journal of Autism and Developmental Disorders, 9, 11-29.

Wood, J. J., Drahota, A., Sze, K., Har, K., Chiu, A., \& Langer, D. A. (2009). Cognitive behavioral therapy for anxiety in children with autism spectrum disorders: A randomized, controlled study. Journal of Child Psychology and Psychiatry, 50, 224-234.

Zandt, F., Prior, M., \& Kyrios, M. (2007). Repetitive behaviour in children with high functioning autism and obsessive compulsive disorder. Journal of Autism and Developmental Disorders, 37, 251-259. 